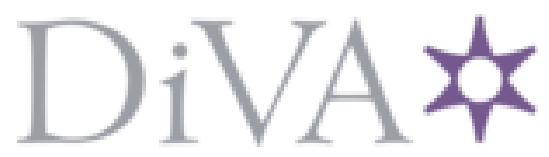

http://www.diva-portal.org

This is the published version of a paper published in Focus on Parkinson's disease.

Citation for the original published paper (version of record):

Sjödahl Hammarlund, C., Hagell, P., Nilsson, M H. (2012)

Motor and non-motor predictors of illness-related distress in Parkinson's disease.

Focus on Parkinson's disease, 23(1): 27-28

Access to the published version may require subscription.

N.B. When citing this work, cite the original published paper.

Permanent link to this version:

http://urn.kb.se/resolve?urn=urn:nbn:se:hkr:diva-14594 


\section{MOTOR AND NON-MOTOR PREDICTORS OF ILLNESS-RELATED DISTRESS IN PARKINSON'S DISEASE}

Deople with Parkinson's disease (PD) suffer from both motor and non-motor symptoms. A recent systematic review concluded that depression, disease severity, and disability negatively influence the patient-reported impact of PD. ${ }^{1}$ However, studies vary in regard to the potentially independent variables included. For example, whereas several studies included depression as a potential contributor to patientreported disease impact, only one study simultaneously included fatigue, depression, and sleep as independent variables while disregarding any motor symptoms. ${ }^{1}$

Our objective was to investigate associations between patient-reported illness-related distress, and motor and non-motor aspects of PD, separately as well as in combination. Data were taken from a cross-sectional sample of 118 people with PD (54\% men; mean age and PD duration 64 and 8 years, respectively; median Hoehn and Yahr
[HY] stage during the "off" phase was III; median Unified PD Rating Scale [UPDRS] motor score during the "on" phase, was 17). Exclusion criteria were: participation in other ongoing studies, infections, psychiatric adverse drug reactions, and clinically significant comorbidities. ${ }^{2}$

Patients were assessed during the "on" phase using the UPDRS, the $\mathrm{HY}$ staging of PD, and the MiniMental State Exam (MMSE). HY stages were also estimated for the

TABLE. MULTIPLE LINEAR REGRESSION MODELS WITH DISTRESS (NHPD SCORE) AS DEPENDENT VARIABLE AMONG PEOPLE WITH PARKINSON'S DISEASEa

\section{Adjusted R ${ }^{2}$}

$\begin{array}{lllll}\text { Significant independent variables }^{\mathrm{b}} & \mathrm{B} & \beta & \mathrm{p} \text { value } & \text { Stepwise change }\end{array}$

Model 1: Non-motor aspects ${ }^{\mathrm{c}}$

$\begin{array}{lrrrrr}\text { Age } & -0.039 & -0.025 & 0.613 & 0.048 & 0.048 \\ \text { Fatigue (FACIT-F) } & 0.468 & 0.310 & <0.001 & 0.473 & 0.521 \\ \text { Pain (NHP Pain) } & 0.212 & 0.364 & <0.001 & 0.153 & 0.674 \\ \text { Sleep quality (PSQI) } & 1.029 & 0.269 & <0.001 & 0.073 & 0.747 \\ \text { Depression (HADS) } & 1.312 & 0.252 & <0.001 & 0.055 & 0.802 \\ \text { Anxiety (HADS) } & 0.473 & 0.119 & 0.047 & 0.006 & 0.808\end{array}$

Model 2: Motor aspects ${ }^{\mathrm{d}}$

Age

Mobility (NHP Mobility)

\section{$-0.028$}

0.357

$$
-0.020
$$

0.577

0.840

$<0.001$

0.037

0.270

0.037

0.307

Model 3: Motor and non-motor aspects ${ }^{\mathrm{e}}$

Age
Fatigue (FACIT-F)
Sleep quality (PSQI)
Pain (NHP pain)
Depression (HADS)

-0.072
0.483
1.031
0.204
1.611

-0.050
0.356
0.234
0.325
0.344

0.026

0.465

0.131

0.055

0.080
0.026

0.491

0.622

0.677

0.757

${ }^{a}$ All models controlled for age (indicated in italics). ${ }^{b}$ Listed by order of entry into the models (forward method).

c Independent variables: time since PD diagnosis (years), cognition (MMSE), fatigue (FACIT-F), daytime sleepiness (ESS), depression (HADS), anxiety (HADS), pain (NHP), sleep quality (PSQI), thought disorder (item 2, UPDRS I, dichotomized; $0=$ no signs of thought disorder, $1=$ signs of thought disorder [scores 1-4]) and motivation (item 4, UPDRS I, dichotomized; 0 = normal motivation, $1=$ impaired motivation [scores 1-4]).

d Independent variables: Parkinsonism (total UPDRS III score), dyskinesias (UPDRS IV), motor fluctuations (UPDRS IV), mobility (NHP), and falling and/or balance problems $(0=$ no, $1=$ yes). Additionally, UPDRS III total score was substituted by symptomatic profile scores (see main text). e Independent variables: as in models 1 and 2.

Reprinted from Parkinsonism Relat Disord. 2012;18:299-302. ${ }^{6}$ Copyright (C) 2011 Elsevier Ltd. 
"off" phase. UPDRS part III (motor score) was used as an overall measure of the severity of Parkinsonian motor symptoms. In addition, the following symptomatic profile scores were calculated: axial/postural/gait impairments, rest tremor, postural tremor, rigidity, and limb bradykinesia. Dyskinesia and motor fluctuation scores were computed from UPDRS part IV (complications of therapy). UPDRS items 2 (thought disorder), 4 (motivation), and 42 (symptomatic orthostatism) were used as coarse indicators of, respectively, psychiatric dopaminergic side-effects, apathy, and dysautonomy. Illness-related distress was assessed using the Nottingham Health Profile index of Distress (NHPD; total score range $0-100$; $100=$ more distress $).{ }^{3}$ Participants also completed the following selfreport rating scales during the "on" phase: the Functional Assessment of Chronic Illness Therapy - Fatigue scale (FACIT-F), the Pittsburgh Sleep Quality Index (PSQI), the Epworth Sleepiness Scale (ESS), the Hospital Anxiety and Depression Scale (HADS), and the Nottingham Health Profile (NHP) Mobility and Pain sections. Participants were also asked whether they had ever experienced balance problems or falls.

Variables significantly associated with NHPD scores in bivariate analyses were entered as independent variables in multiple linear regression models with distress (NHPD) as the dependent variable. To ease interpretation, all scores were adjusted to be in the same direction (higher scores $=$ more problems). Three main regression models were estimated: in model 1 , only independent variables representing non-motor aspects were included, while in model 2 only motor variables were included, and model 3 included both motor and non-motor aspects as independent variables.

Our results revealed five significant associations between non-motor aspects of $\mathrm{PD}$ and illness-reported distress (model 1), accounting for $81 \%$ of the variance in NHPD scores (Table; see cumulative adjusted $\mathrm{R}^{2}$ values). Only one significant independent variable (self-reported mobility) was identified when only motor aspects of PD were considered (model 2). Controlling for age, this variable accounted for $31 \%$ of the variance in NHPD scores. Using the five UPDRS III motor symptom profile scores as independent variables, instead of the total UPDRS III motor score, did not change these results. Using only UPDRS-derived motor variables (the five UPDRS III motor symptom profile scores, dyskinesia, and motor fluctuation scores) resulted in one independent variable (axial/postural/ gait score) significantly associated with distress (B, 0.93), accounting for 8.4\% of the variance in distress scores (data not shown). Exploring associations with illness-related distress from a broad range of both motor and non-motor aspects of PD (model 3) identified four significant independent variables, accounting for $76 \%$ of the variance in NHPD scores (Table). The strongest association was found for fatigue, followed by depression, pain, and sleep quality.

These observations imply that illness-related distress in PD appears to be mainly influenced by non-motor disease aspects (e.g. fatigue, sleep, and pain), while a main motor component is that of mobility rather than the underpinning PD motor symptoms per se. Fatigue contributed the most to the variance in distress scores, which highlights the importance of this aspect from the patient's viewpoint. These observations are in agreement with previous data, ${ }^{1}$ but our results are unique in that they have confirmed these associations in a single sample in which the potential impact of both motor and non-motor aspects of PD are considered, individually as well as in combination. As such, the present findings suggest that non-motor features of PD are of utmost importance, and that mobility-related problems constitute the primary motor component of the impact of PD from the perspective of the patient. This finding underscores the need to take these aspects into consideration in clinical practice and research. Specifically, there is a need for interventions targeting non-motor symptoms (e.g. fatigue, pain, sleep, and depression) and mobility (e.g. gait and balance impairment). Such interventions are of particular importance because common dopaminergic medications often have limited effects on these symptoms. Indeed, aspects identified as potential contributors to distress in this study mainly appear related to non-dopaminergic lesions, 4,5 which has implications in terms of therapeutic priorities in order to minimize the impact of the disease on people with PD.

Sjödahl Hammarlund S, Hagell P, Nilsson $\mathrm{MH}$. Motor and non-motor predictors of illness-related distress in Parkinson's disease. Parkinsonism Relat Disord. 2012;18:299-302.

Correspondence: Peter Hagell, School of Health and Society, Kristianstad University, SE-29188 Kristianstad, Sweden. Email: Peter.Hagell@hkr.se

\section{REFERENCES}

1. Soh SE, Morris ME, McGinley JL. Determinants of health-related quality of life in Parkinson's disease: a systematic review. Parkinsonism Relat Disord. 2011;17:1-9.

2. Hagell P, Höglund A, Reimer J, et al. Measuring fatigue in Parkinson's disease: a psychometric study of two brief generic fatigue questionnaires. J Pain Symptom Manage. 2006;32:420-32.

3. McKenna S, Hunt S, Tennant A. The development of a patient-completed index of distress from the Nottingham Health Profile: A new measure for use in cost utility studies. Br J Med Econ. 1993;6:13-24.

4. Grimbergen YA, Langston JW, Roos RA, et al. Postural instability in Parkinson's disease: the adrenergic hypothesis and the locus coeruleus. Expert Rev Neurother. 2009;9:279-90.

5. Pavese N, Metta V, Bose SK, et al. Fatigue in Parkinson's disease is linked to striatal and limbic serotonergic dysfunction. Brain. 2010;133:3434-43. 